\title{
The Structure of a Chronic Total Occlusion and Its Safe Treatment in a Patient with Chronic Thromboembolic Pulmonary Hypertension
}

\author{
Shun Minatsuki, ${ }^{1} \mathrm{MD}$, Masaru Hatano, ${ }^{2} \mathrm{MD}$, Hisataka Maki, ${ }^{1} \mathrm{MD}$, \\ Jiro Ando, ${ }^{1} \mathrm{MD}$ and Issei Komuro, ${ }^{1} \mathrm{MD}$
}

\begin{abstract}
Summary
A pouching defect, which is a type of angiographic classification in chronic thromboembolic pulmonary hypertension (CTEPH), is equivalent to a chronic total occlusion (CTO). Thus far, treating CTO involves high risk of the lung bleeding and is difficult because the structure and treating strategy have not been clarified, yet treating it has great potential to improve hemodynamic status and ventilation perfusion mismatch. Here, we describe a case of successfully treated the CTO. In this case, we treated it safely by two balloon pulmonary angiography (BPA) sessions. In first session, the surface of the CTO was partially broken by the catheter. However, the distal vessels of it were not observed. Pulmonary angiogram was performed 4 months later, the distal vessels of CTO were observed. This lesion was no longer the CTO, we performed BPA safely and pulmonary arterial pressure was improved dramatically. Furthermore, pulmonary angiogram revealed the surface of the CTO has a cap which is comprised of cross-aggregation of organized thrombi. Partial breakage of the cap is a key to recanalization of the CTO, even if the distal vessels were not seen in same BPA session.
\end{abstract}

(Int Heart J 2017; 58: 824-827)

Key words: Balloon pulmonary angioplasty, Total occlusion

$\mathrm{C}$ hronic thromboembolic pulmonary hypertension (CTEPH) is a type of pulmonary hypertension that occurs when blood flow is restricted by organized thrombi in the pulmonary artery. Pulmonary endarterectomy is the initial treatment for CTEPH. However, balloon pulmonary angioplasty (BPA) and medicinal therapy are considered for inoperative cases of CTEPH. During BPA, blood flow is improved by compressing the organized thrombi into the vascular wall and dilating the external diameter of the pulmonary artery by inflating the balloon to the optimal size. The angiographic classification is very important to determine the optimal size of the balloon. ${ }^{1)}$ A pouching defect, which is a type of angiographic classification, is equivalent to a chronic total occlusion (CTO). There are usually numerous vessels distal to a CTO. Thus, the treatment of a CTO has the potential to dramatically improve the patient's hemodynamic status; however, treatment is very difficult due to anatomic issues and to high risk of the lung bleeding. The success rate of performing BPA to treat a CTO is up to $50 \%$ even at centers that specialize in performing BPA. ${ }^{1)}$ This is because, the precise structure of a CTO and a detailed strategy for safely treating CTOs are unclear.

\section{Case Report}

We describe the case of a 66-year-old woman with inoperative CTEPH who successfully and safely underwent BPA for the treatment of a CTO. Pulmonary angiography (PAG) findings showed a CTO in the right lung (Figure 1A); however, even after the catheter was pressed on the surface of the CTO to observe the distal vessel, we could not observe the distal vessel on angiography (Figure 1B). Therefore, we did not perform BPA at this time due to the high risk of the lung bleeding involved. Follow-up PAG evaluation was performed 4 months later, and the results showed the distal vessel that had not been observed previously (Figure 1C). Furthermore, a cap consisting of cross-aggregation of organized thrombi with a few micro channels and the aggregation of organized thrombi distal to the cap were observed (Figure 1D). This lesion was no longer a CTO, as it became a complete obstruction; thus, we performed BPA.

At the time of BPA, intravascular ultrasound (IVUS) findings showed that the organized thrombi were densely aggregated (Figure 2A), and there were a few blood flow signals presented in the microchannel of the organized thrombi on the ChromaFlo ${ }^{\circledR}$ image (Eagle Eye Gold, Volcano Therapeutics), which is an imaging tool used to

From the ${ }^{1}$ Department of Cardiovascular Medicine, Graduate School of Medicine, The University of Tokyo, Tokyo, Japan and ${ }^{2}$ Department of Therapeutic Strategy for Heart Failure, Graduate School of Medicine, The University of Tokyo, Tokyo, Japan.

Address for correspondence: Shun Minatsuki, MD, Department of Cardiovascular Medicine, Graduate School of Medicine, The University of Tokyo, 7-3-1 Hongo, Bunkyo-ku, Tokyo 113-8655, Japan. E-mail: minatsuki-tky@umin.ac.jp

Received for publication October 3, 2016. Revised and accepted November 22, 2016.

Released in advance online on J-STAGE September 30, 2017.

doi: 10.1536/ihj.16-494

All rights reserved by the International Heart Journal Association. 

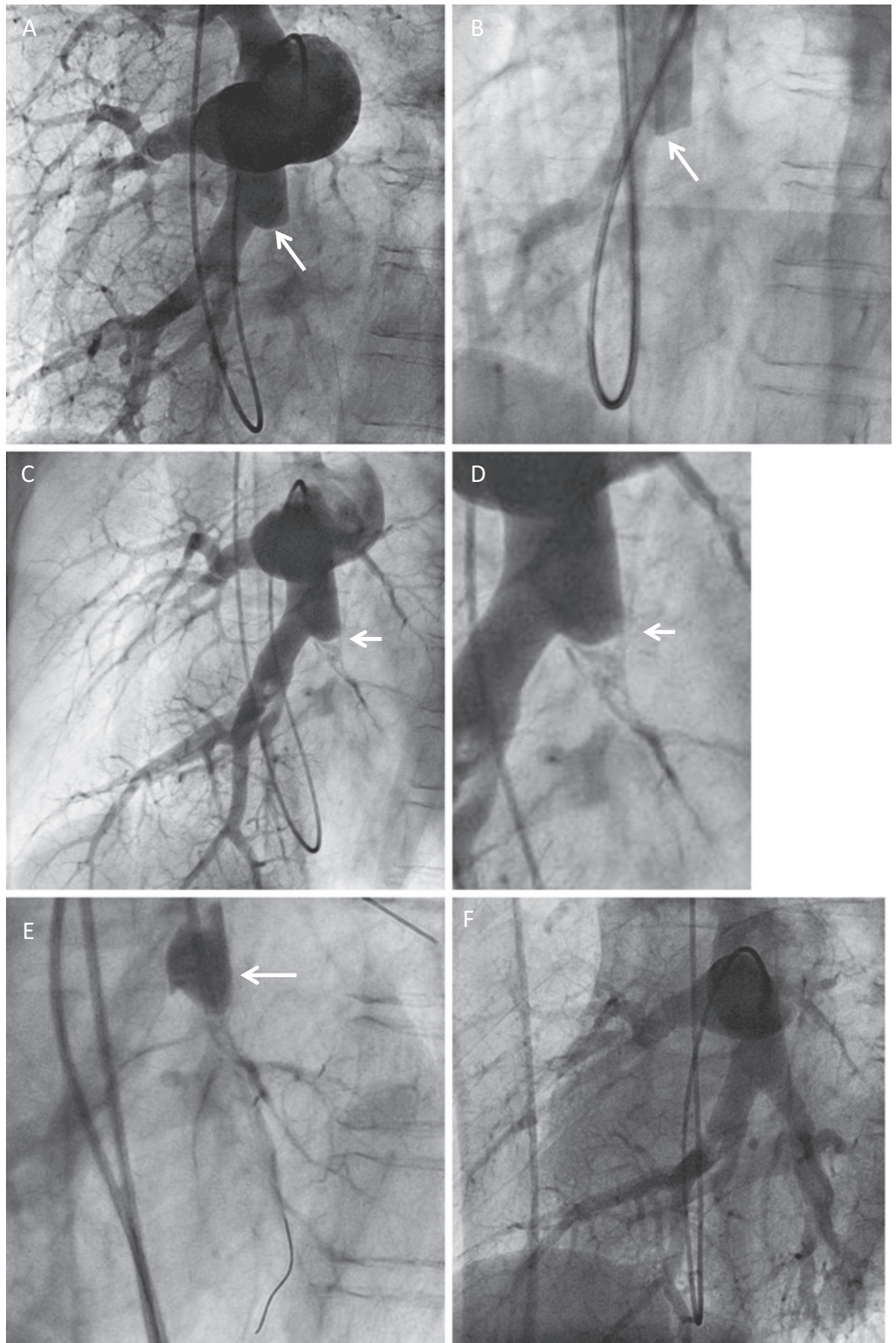

Figure 1. Pulmonary angiography images showing the posterior basal segment of the right lung. A: Pulmonary angiogram image of the chronic total occlusion (CTO) (arrow). B: Pulmonary angiogram image of the catheter was pressed on the surface of the CTO (arrow). C-D: Follow-up angiogram image showing the cap (arrow) and recanalization of the CTO (C: normal range; D: magnified range). E: Angiogram image from the catheter showing the stagnation of contrast medium between the cap and the superficial layer of the cap (arrow). F: After balloon pulmonary angioplasty was performed, the distal vessels were clearly seen.

visualize blood flow (Figure 2B). The red signal existing the external usually indicates the flow of pulmonary vein. Angiography results demonstrated stagnation of the contrast medium adjacent to the cap, specifically between the layer of organized thrombi and the cap (Figure 1E). We safely performed BPA and recanalization (Figure 1F). After recanalization, IVUS results showed compressed organized thrombi on the vessel wall, and the external diameter of the vessel was dilated (Figure 2C). The ChromaFlo ${ }^{\circledR}$ image also indicated an increase in the blood 

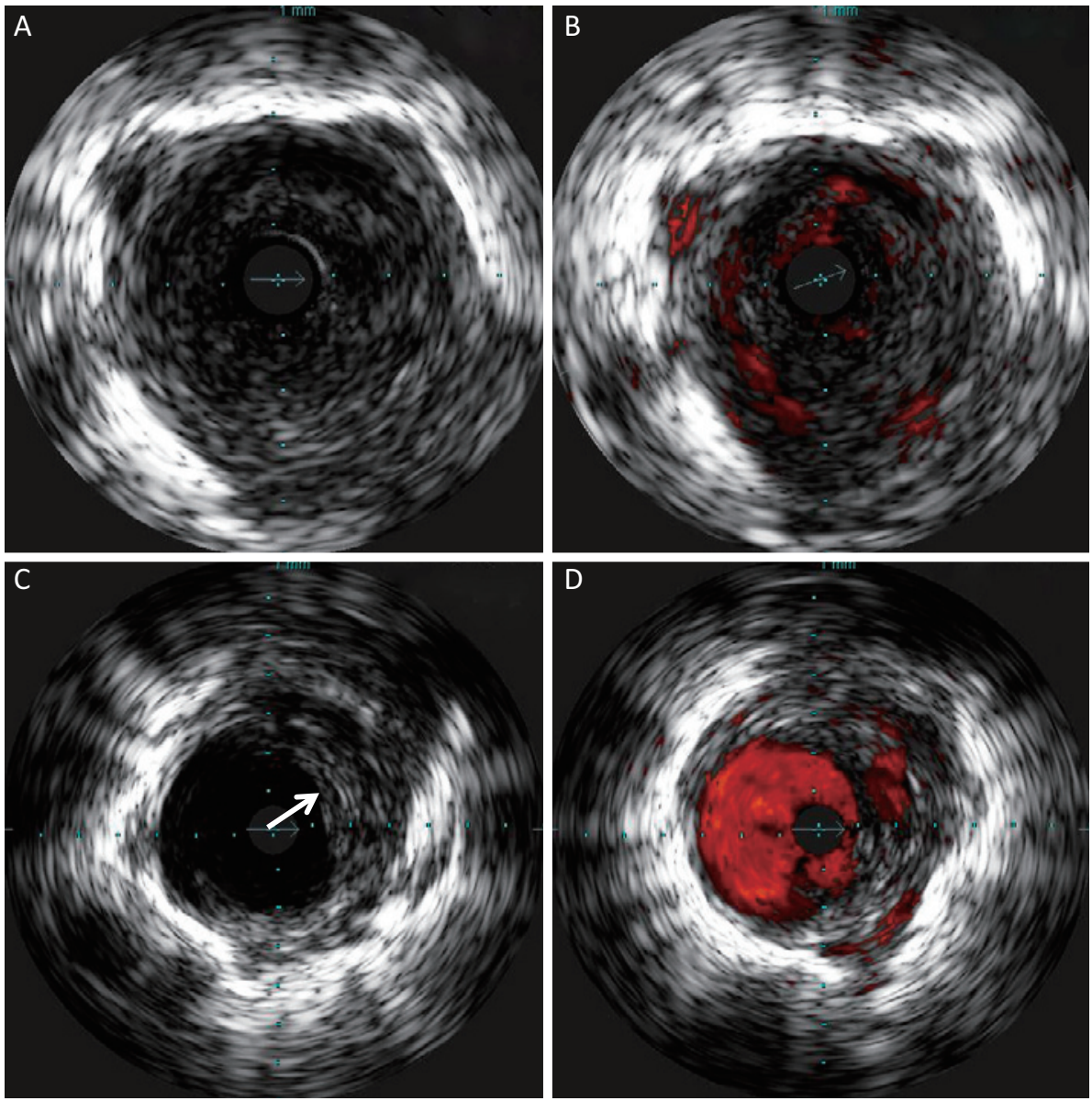

Figure 2. Intravascular ultrasound image of the chronic total occlusion before and immediately after balloon dilatation was performed. A, B: Images before dilatation (A: normal image; B: Chromaflow ${ }^{\circledR}$ image) showing the densely aggregated, organized thrombi. A few red signals were seen where the microchannel of organized thrombi. C, D: Images immediately after dilatation was performed with a 4-mm diameter balloon (C: normal image; D: Chromaflow ${ }^{\circledR}$ image). Blood flow was improved by compressing the organized thrombi to the vessel wall (arrow), and the external diameter of the vessel was expanded compared to that before dilatation.

flow, where the organized thrombi were dilated by balloon (Figure 2D). Her mean pulmonary artery pressure was markedly improved immediately after treated CTO $(31 \rightarrow$ $24 \mathrm{mmHg})$.

\section{Discussion}

The effectiveness and safety of using BPA to treat CTEPH has been reported. ${ }^{2,3)}$ Treating a CTO is difficult, because the distal vessels cannot be observed on an angiogram. When the guidewire crosses the cap distally, it is unclear whether the tip of the wire is inside of the vessel. If the tip is outside of the pulmonary artery, lung bleeding, which sometimes can become a life-threatening complication, can occur. Furthermore, the organized thrombi of a cap are thick, so we usually use a heavier weighted loading wire to cross the cap. The risk of penetrating the lung is higher with treating a CTO than treating other types of lesions.

The key for devising a strategy to safely treat a CTO with BPA is to understand the detailed structure of a CTO. The cap and aggregation of organized thrombi distal to the cap may be the main etiologic components of a CTO. IVUS imaging showed that the vessel of the CTO was comprised of a large amount of organized thrombi, especially in the cap, and the diameter of the distal vessels was significantly decreased due to the lack of blood flow. In our case, the superficial layer of the cap may have been torn off previously after the catheter was pressed on the cap or after the contrast medium was injected into the cap. This made small route to distal vessel and blood gradually flowed into the distally located organized thrombi, and the CTO was recanalized. Thus, partial breakage of the cap by using a catheter or guidewire initially and making small route to distal vessel can be a safe alternative strategy for treating CTOs. By using IVUS imaging, surgeons can know whether the crossed guidewire is in the vessels before balloon dilatation is performed.

At our institution, 6 of 8 cases with a CTO were suc- 
cessfully treated with BPA without complications. However, to ensure safety, a two-staged treatment can be performed: first, break the cap to make small route to distal vessel; and second, perform BPA when the distal vessels are visible. This is one of the methods for safely performing BPA to treat a CTO.

\section{Disclosures}

Conflict of interest: None.

\section{References}

1. Ogawa A, Matsubara H. Balloon pulmonary angioplasty: a treatment option for inoperable patients with chronic thromboembolic pulmonary hypertension. Front Cardiovasc Med 2015; 2: 4.

2. Mizoguchi H, Ogawa A, Munemasa M, Mikouchi H, Ito H, Matsubara H. Refined balloon pulmonary angioplasty for inoperable patients with chronic thromboembolic pulmonary hypertension. Circ Cardiovasc Interv 2012; 5: 748-55.

3. Andreassen AK, Ragnarsson A, Gude E, Geiran O, Andersen R. Balloon pulmonary angioplasty in patients with inoperable chronic thromboembolic pulmonary hypertension. Heart 2012; 99: $1415-20$ 